\title{
Longevity of Purebred Dog Breeds
}

\section{Evžen Korec*}

Department of Genetics, ZOO Tábor, Czech Republic

*Corresponding author: Evžen Korec, Department of Genetics, Z0O Tábor, Dukelských hrdinů 19, Prague 7, 17000, Czech Republic

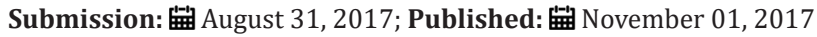

\begin{abstract}
Body mass is a very significant factor for influencing longevity. Generally, large animal species tend to live longer than small species. In domestic dogs, the relationship between body size and lifespan shows opposite trend and increasing bodyweight is negatively correlated with longevity. Crossbred dogs have increased longevity, compared with purebreds. In the Cane Corso Italiano breed, a relationship between longevity and hair colour was found for the first time in mammals.

Understanding longevity and aging across species and individuals is critical for reaching higher ages at death in animals, as well as in humans. By understanding genetics, the age limits of animals and humans can be significantly prolonged. Detection and analysis of genes associated with longevity present a very promising method for prolonging life.

Body mass is a very significant factor for influencing longevity. Generally, large animal species tend to live longer than small species [1,2]. This rule has some exceptions. In domestic dogs, the relationship between body size and lifespan shows the opposite trend, and increasing bodyweight is negatively correlated with longevity [3,4]. A negative correlation has also been observed between height and longevity [5]. The lifespan of most dog breeds has already been described in the past [3,4]. Cane Corso is the last dog breed in which their lifespan was determined [6]. Breed - specific age at death is shown in Table 1.
\end{abstract}

Table 1: Longevity of Purebred Dog Breeds.

\begin{tabular}{|c|c|c|c|}
\hline Breed Name & Median Longevity (Years) & Breed Weight Group** & Source \\
\hline Affen pinscher & 11,42 & 2 & Adams (2010) \\
\hline Afghan hound & 11,92 & 4 & Adams (2010) \\
\hline Airedale terrier & 10,75 & 3 & Adams (2010) \\
\hline Akita & 9,92 & 4 & Adams (2010) \\
\hline Alaskan malamute & 10,71 & 3 & Adams (2010) \\
\hline American cocker spaniel & 10,33 & 3 & Adams (2010) \\
\hline Anatolian/Karabash & 10,75 & 4 & Adams (2010) \\
\hline Australian cattle dog & 11,67 & 3 & Adams (2010) \\
\hline Australian shepherd & 9 & 3 & Adams (2010) \\
\hline Australian silky terrier & 14,25 & 1 & Adams (2010) \\
\hline Australian terrier & 12,08 & 1 & Adams (2010) \\
\hline Basenji & 13,54 & 2 & Adams (2010) \\
\hline Basset Fauve de Bretagne & 10,42 & 3 & Adams (2010) \\
\hline Basset griffon vendeen & 12,04 & 3 & Adams (2010) \\
\hline Basset hound & 11,29 & 3 & Adams (2010) \\
\hline Beagle & 12,67 & 2 & Adams (2010) \\
\hline Bearded collie & 13,5 & 3 & Adams (2010) \\
\hline Bearded collie & 13,7 & 3 & O’Neil (2013) \\
\hline Bedlington terrier & 13,38 & 2 & Adams (2010) \\
\hline Belgian shepherd & 12,5 & 4 & Adams (2010) \\
\hline Bernese mountain dog & 8 & 4 & Adams (2010) \\
\hline Bichon frise & 12,92 & 1 & Adams (2010) \\
\hline
\end{tabular}




\begin{tabular}{|c|c|c|c|}
\hline Breed Name & Median Longevity (Years) & Breed Weight Group** & Source \\
\hline Bichon frise & 12,7 & 1 & O’Neil (2013) \\
\hline Bloodhound & 6,79 & 5 & Adams (2010) \\
\hline Border collie & 12,25 & 3 & Adams (2010) \\
\hline Border collie & 13,5 & 3 & O’Neil (2013) \\
\hline Border terrier & 14 & 2 & Adams (2010) \\
\hline Border terrier & 12 & 2 & O’Neil (2013) \\
\hline Borzoi & 9,08 & 4 & Adams (2010) \\
\hline Boston terrier & 10,92 & 2 & Adams (2010) \\
\hline Bouvier des flandres & 11,33 & 4 & Adams (2010) \\
\hline Boxer & 10,25 & 4 & Adams (2010) \\
\hline Boxer & 10 & 4 & O’Neil (2013) \\
\hline Briard & 11,17 & 4 & Adams (2010) \\
\hline Brittany & 12,88 & 3 & Adams (2010) \\
\hline Bull terrier & 10 & 3 & Adams (2010) \\
\hline Bull terrier & 11,2 & 3 & O’Neil (2013) \\
\hline Bulldog & 6,29 & 3 & Adams (2010) \\
\hline Bulldog & 8,4 & 3 & O’Neil (2013) \\
\hline Bullmastiff & 7,46 & 4 & Adams (2010) \\
\hline Cairn terrier & 14 & 2 & Adams (2010) \\
\hline Cairn terrier & 13,4 & 2 & O’Neil (2013) \\
\hline Canaan dog & 14,63 & 3 & Adams (2010) \\
\hline Cane corso italiano & 9,29 & 4 & Korec (2017) \\
\hline Cavalier king charles spaniel & 11,38 & 2 & Adams (2010) \\
\hline Cavalier king charles spaniel & 9,9 & 2 & O’Neil (2013) \\
\hline Cesky terrier & 8,42 & 2 & Adams (2010) \\
\hline Clumber spaniel & 10,33 & 4 & Adams (2010) \\
\hline Cocker spaniel & 11,17 & 3 & Adams (2010) \\
\hline Cocker spaniel & 11,5 & 3 & O’Neil (2013) \\
\hline Collie & 12,67 & 3 & Adams (2010) \\
\hline Curly coated retriever & 10,75 & 4 & Adams (2010) \\
\hline Dachshund & 12,67 & 2 & Adams (2010) \\
\hline Dalmatian & 12,5 & 4 & Adams (2010) \\
\hline Dalmatian & 13,3 & 4 & O’Neil (2013) \\
\hline Dandie Dinmont terrier & 12,17 & 2 & Adams (2010) \\
\hline Deerhound & 8,67 & 5 & Adams (2010) \\
\hline Dobermann & 9,2 & 4 & O’Neil (2013) \\
\hline Dobermann & 10,5 & 4 & Adams (2010) \\
\hline Dogue de Bordeaux & 3,83 & 4 & Adams (2010) \\
\hline Dogue de Bordeaux & 5,5 & 4 & O’Neil (2013) \\
\hline English setter & 11,58 & 4 & Adams (2010) \\
\hline English springer spaniel & 12 & 3 & Adams (2010) \\
\hline English springer spaniel & 13,3 & 3 & O’Neil (2013) \\
\hline English toy terrier & 12 & 1 & Adams (2010) \\
\hline Estrela mountain & 9,75 & 3 & Adams (2010) \\
\hline Field spaniel & 11,63 & 3 & Adams (2010) \\
\hline Finnish lapphund & 7,33 & 2 & Adams (2010) \\
\hline Finnish spitz & 11,13 & 3 & Adams (2010) \\
\hline
\end{tabular}




\begin{tabular}{|c|c|c|c|}
\hline Breed Name & Median Longevity (Years) & Breed Weight Group** & Source \\
\hline Flat-coated retriever & 9,83 & 4 & Adams (2010) \\
\hline Fox terrier & 13,13 & 2 & Adams (2010) \\
\hline French bulldog & 9 & 2 & Adams (2010) \\
\hline German longhaired pointer & 10,5 & 3 & Adams (2010) \\
\hline German pinscher & 11,38 & 2 & Adams (2010) \\
\hline German shepherd dog & 11 & 4 & O’Neil (2013) \\
\hline German shorthaired pointer & 12 & 4 & Adams (2010) \\
\hline German spitz & 11,33 & 2 & Adams (2010) \\
\hline German wirehaired pointer & 10 & 4 & Adams (2010) \\
\hline Giant schnauzer & 10 & 4 & Adams (2010) \\
\hline Glen of imaal terrier & 10,42 & 3 & Adams (2010) \\
\hline Golden retriever & 12,25 & 4 & Adams (2010) \\
\hline Golden retriever & 12,5 & 4 & O’Neil (2013) \\
\hline Gordon setter & 11,08 & 3 & Adams (2010) \\
\hline Grand bleu de gascoigne & 4,54 & 3 & Adams (2010) \\
\hline Great dane & 6,5 & 5 & Adams (2010) \\
\hline Great dane & 6 & 5 & O'Neil (2013) \\
\hline Greenland dog & 8,46 & 3 & Adams (2010) \\
\hline Grey hound & 9,08 & 4 & Adams (2010) \\
\hline Grey hound & 10,8 & 4 & O’Neil (2013) \\
\hline Griffon bruxellois & 12 & 1 & Adams (2010) \\
\hline Hamilton stovare & 10,13 & 3 & Adams (2010) \\
\hline Havanese & 10,25 & 3 & Adams (2010) \\
\hline Hovawart & 12,92 & 3 & Adams (2010) \\
\hline Hungarian puli & 12,42 & 3 & Adams (2010) \\
\hline Hungarian vizsla & 12,92 & 4 & Adams (2010) \\
\hline Hungarian wirehaired vizsla & 9,83 & 3 & Adams (2010) \\
\hline Chesapeake bay retriever & 10,75 & 3 & Adams (2010) \\
\hline Chihuahua & 12,42 & 1 & Adams (2010) \\
\hline Chihuahua & 7,1 & 1 & O’Neil (2013) \\
\hline Chinese crested & 10,08 & 1 & Adams (2010) \\
\hline Chow chow & 9,38 & 3 & Adams (2010) \\
\hline Irish red \& white setter & 11,42 & 3 & Adams (2010) \\
\hline Irish setter & 12 & 4 & Adams (2010) \\
\hline Irish terrier & 14,83 & 2 & Adams (2010) \\
\hline Irish water spaniel & 9,33 & 4 & Adams (2010) \\
\hline Irish wolfhound & 7,04 & 5 & Adams (2010) \\
\hline Italian greyhound & 13,5 & 1 & Adams (2010) \\
\hline Italian spinone & 9 & 4 & Adams (2010) \\
\hline Jack russell terrier & 13,4 & 2 & O’Neil (2013) \\
\hline Japanese chin & 9,25 & 1 & Adams (2010) \\
\hline Japanese spitz & 12,29 & 1 & Adams (2010) \\
\hline Keeshond & 12,21 & 3 & Adams (2010) \\
\hline Kerry Blue terrier & 11,5 & 3 & Adams (2010) \\
\hline King charles spaniel & 10,04 & 2 & Adams (2010) \\
\hline King charles spaniel & 12 & 2 & O'Neil (2013) \\
\hline Komondor & 9,13 & 5 & Adams (2010) \\
\hline
\end{tabular}




\begin{tabular}{|c|c|c|c|}
\hline Breed Name & Median Longevity (Years) & Breed Weight Group** & Source \\
\hline Kooikerhondje & 3,92 & 3 & Adams (2010) \\
\hline Labrador retriever & 12,25 & 4 & Adams (2010) \\
\hline Labrador retriever & 12,5 & 4 & O’Neil (2013) \\
\hline Lakeland terrier & 15,46 & 2 & Adams (2010) \\
\hline Lancashire heeler & 11,75 & 2 & Adams (2010) \\
\hline Large munsterlander & 11,33 & 4 & Adams (2010) \\
\hline Leonberger & 7,08 & 5 & Adams (2010) \\
\hline Lhasa apso & 13 & 2 & O’Neil (2013) \\
\hline Lhasa apso & 14,33 & 2 & Adams (2010) \\
\hline Lowchen & 10 & 1 & Adams (2010) \\
\hline Maltese & 12,25 & 1 & Adams (2010) \\
\hline Manchester terrier & 12,83 & 1 & Adams (2010) \\
\hline Maremma sheepdog & 10 & 4 & Adams (2010) \\
\hline Mastiff & 6,83 & 5 & Adams (2010) \\
\hline Mastiff & 7,1 & 5 & O’Neil (2013) \\
\hline Miniature bull terrier & 6,08 & 2 & Adams (2010) \\
\hline Miniature dachshund & 13,5 & 1 & O’Neil (2013) \\
\hline Miniature pinscher & 13 & 1 & Adams (2010) \\
\hline Miniature poodle & 13,92 & 2 & Adams (2010) \\
\hline Miniature poodle & 14,2 & 2 & O’Neil (2013) \\
\hline Miniature schnauzer & 12,08 & 2 & Adams (2010) \\
\hline Neopolitan mastiff & 2,33 & 4 & Adams (2010) \\
\hline Newfoundland & 9,67 & 5 & Adams (2010) \\
\hline Norfolk terrier & 11 & 2 & Adams (2010) \\
\hline Norwegian buhund & 12,67 & 2 & Adams (2010) \\
\hline Norwegian elkhound & 13,17 & 3 & Adams (2010) \\
\hline Norwich terrier & 13,38 & 2 & Adams (2010) \\
\hline Nova Scotia duck tolling retriever & 8 & 3 & Adams (2010) \\
\hline Old english sheepdog & 10,75 & 4 & Adams (2010) \\
\hline Otterhound & 10,21 & 4 & Adams (2010) \\
\hline Papillon/Butterfly dog & 13,08 & 1 & Adams (2010) \\
\hline Parson russell terrier & 13 & 2 & Adams (2010) \\
\hline Pekingese & 11,42 & 1 & Adams (2010) \\
\hline Pharoah hound & 11,83 & 3 & Adams (2010) \\
\hline Pointer & 12,42 & 3 & Adams (2010) \\
\hline Polish lowland sheepdog & 9,58 & 3 & Adams (2010) \\
\hline Pomeranian & 9,67 & 1 & Adams (2010) \\
\hline Portuguese water dog & 11,42 & 3 & Adams (2010) \\
\hline Pug & 11 & 2 & Adams (2010) \\
\hline Pyrenean mountain dog & 9,58 & 5 & Adams (2010) \\
\hline Pyrenean sheepdog & 5,79 & 4 & Adams (2010) \\
\hline Rhodesian ridgeback & 11 & 4 & Adams (2010) \\
\hline Rottweiler & 8,92 & 4 & Adams (2010) \\
\hline Rottweiler & 8 & 4 & O’Neil (2013) \\
\hline Rough collie & 12 & 3 & O’Neil (2013) \\
\hline Russian black terrier & 1,79 & 3 & Adams (2010) \\
\hline Saluki/gazelle hound & 12 & 4 & Adams (2010) \\
\hline
\end{tabular}




\begin{tabular}{|c|c|c|c|}
\hline Breed Name & Median Longevity (Years) & Breed Weight Group** & Source \\
\hline Samoyed & 12,5 & 4 & Adams (2010) \\
\hline Scottish terrier & 10,25 & 2 & Adams (2010) \\
\hline Scottish terrier & 12 & 2 & O’Neil (2013) \\
\hline Sealyham terrier & 12,25 & 2 & Adams (2010) \\
\hline Shar pei & 6,29 & 3 & Adams (2010) \\
\hline Shetland sheepdog & 12,5 & 2 & Adams (2010) \\
\hline Shetland sheepdog & 12,5 & 2 & O’Neil (2013) \\
\hline Shiba Inu (Japanese) & 7 & 2 & Adams (2010) \\
\hline Shih-tzu & 13,17 & 2 & Adams (2010) \\
\hline Shih-tzu & 13,3 & 2 & O’Neil (2013) \\
\hline Schipperke & 13 & 2 & Adams (2010) \\
\hline Schnauzer (standard) & 11,96 & 3 & Adams (2010) \\
\hline Siberian husky & 12,58 & 3 & Adams (2010) \\
\hline Skye terrier & 11 & 2 & Adams (2010) \\
\hline Soft coated wheaten terrier & 12,5 & 3 & Adams (2010) \\
\hline St Bernard & 7 & 5 & Adams (2010) \\
\hline Staffordshire bull terrier & 10,7 & 3 & O'Neil (2013) \\
\hline Staffordshire bull terrier & 12,75 & 3 & Adams (2010) \\
\hline Standard poodle & 12 & 4 & Adams (2010) \\
\hline Sussex spaniel & 11,13 & 3 & Adams (2010) \\
\hline Swedish vallhund & 14,42 & 2 & Adams (2010) \\
\hline Tibetan mastiff & 11,92 & 4 & Adams (2010) \\
\hline Tibetan spaniel & 14,42 & 2 & Adams (2010) \\
\hline Tibetan terrier & 12,17 & 2 & Adams (2010) \\
\hline Toy poodle & 14,63 & 1 & Adams (2010) \\
\hline Weimaraner & 11,13 & 4 & Adams (2010) \\
\hline Weimaraner & 12,6 & 4 & O’Neil (2013) \\
\hline Welsh corgi & 12,21 & 2 & Adams (2010) \\
\hline Welsh springer spaniel & 12,58 & 3 & Adams (2010) \\
\hline Welsh terrier & 12,67 & 2 & Adams (2010) \\
\hline West highland white terrier & 13 & 2 & Adams (2010) \\
\hline West highland white terrier & 13,5 & 2 & O’Neil (2013) \\
\hline Whippet & 12,79 & 2 & Adams (2010) \\
\hline Yorkshire terrier & 12,67 & 1 & Adams (2010) \\
\hline Yorkshire terrier & 13 & 1 & O’Neil (2013) \\
\hline
\end{tabular}

** Breed Weight Group: 1 = toy, $2=$ small, $3=$ medium, 4 = large, 5 = giant

Crossbred dogs have increased longevity, compared with purebreds, irrespective of bodyweight, based on predicted effects from hybrid vigour. Crossbreds demonstrated a 1.2 year average survival advantage over purebreds [4]. A valuable study of dog breeds compared purebred and crossbred longevity across five bodyweight categories, which demonstrated that age at death for purebred dogs was significantly lower than that of crossbred dogs for each bodyweight group [7]. This finding suggests that hybrid vigour substantially affects longevity in dogs. One possible explanation is that hybrid dogs are less likely to be homozygous for deleterious genes [8]. Only molecular genetic analysis of genes associated with longevity can explain this feature.
Young dogs usually died from gastrointestinal and infections causes, whereas older dogs died of neurological and neoplastic

causes [9]. Breed specific proportional mortalities were described as the most common causes of death in 72 breeds. The breeds with the highest proportional mortalities for cancer included, in descending order, Irish water spaniel, Flat-coated retriever, Hungarian wirehaired vizsla, Bernese mountain dog, Rottweiler, Italian spinone, Leonberger, Staffordshire bullterrier, Welsh terrier, and Giant schnauzer [3]. Breeds with a cardiac condition as the highest breed specific proportional mortality, in descending order, included Cavalier King Charles spaniel, Norfolk terrier, Deerhound, 
Griffon Bruxellois, and the British bulldog [3]. In the Cane Corso Italiano breed, a relationship between longevity and hair colour was found for the first time in mammals [6]. This finding can suggest some relationship between genes associated with longevity and genes responsible for hair colour.

\section{Conclusion}

Understanding longevity and aging across species and individuals is critical for reaching higher ages at death in animals, as well as in humans. By understanding genetics, the age limits of animals and humans can be significantly prolonged. Detection and analysis of genes associated with longevity present a very promising method for prolongation life. In the Cane Corso Italiano dog breed a relationship between longevity and hair colour was found for the first time in mammals.

\section{References}

1. Galis F, Van Der Sluijs I, Van Dooren TJM, Metz JAJ, Nussbaumer M (2007) Do large dogs die young? J Exp Zool Part B: Molecular and Developmental Evolution 308(2): 119-126.
2. Austad SN (2010) Cats, 'rats', and bats: the comparative biology of aging in the $21^{\text {st }}$ century. Integr Comp Biol 50(5): 783-792.

3. Adams VJ, Evans KM, Sampson J, Wood JLN (2010) Methods and mortality results of a health survey of purebred dogs in the UK. J Small Anim Pract 51(10): 512-524.

4. O'Neill DG, Church DB, McGreevy PD, Thomson PC, Brodbelt DC (2013) Longevity and mortality of owned dogs in England. Vet J 198(3): 638643.

5. Greer KA, Canterberry SC, Murphy KE (2007) Statistical analysis regarding the effects of height and weight on life span of the domestic dog. Res Vet Sci 82(2): 208-214.

6. Korec E, Chalupa O, Hančl M, Korcová J, Bydžovská M (2017) Longevity of Cane Corso Italiano dog breed and its relationship with hair colour. Open Vet J 7(2): 170-173.

7. Patronek GJ, Waters DJ, Glickman LT (1997) Comparative longevity of pet dogs and humans: Implications for gerontology research. J Gerontol A Biol Sci Med Sci 52(3): 171-178.

8. McGreevy PD, Nicholas FW (1999) Some practical solutions to welfare problems in dog breeding. Anim Welf 8: 329-341.

9. Fleming JM, Creevy KE, Promislow DEL (2011) Mortality in North American dogs from 1984 to 2004: An investigation into age- size- and breed-related causes of death. J Vet Inter Med 25: 187-198. 